\title{
Inclusion Of Perineural Invasion With AJCC-TNM Staging: Outcomes From A South East Asian Cohort Of Curatively Treated Gastric Cancer Patients
}

Dillip Kumar Muduly ( $\square$ dillipmuduly@gmail.com )

All India Institute of Medical Sciences - Bhubaneswar https://orcid.org/0000-0001-6427-5714

Madhabananda Kar

All India Institute of Medical Sciences - Bhubaneswar

Mahesh Sultania

All India Institute of Medical Sciences - Bhubaneswar https://orcid.org/0000-0003-1008-8694

Mohammed Shahin

All India Institute of Medical Sciences - Bhubaneswar

\section{Susama Patra}

All India Institute of Medical Sciences - Bhubaneswar

\section{Viswajeet Singh}

All India Institute of Medical Sciences, New Delhi

\section{Mohammed Imaduddin}

All India Institute of Medical Sciences - Bhubaneswar

Hemant Nayak

All India Institute of Medical Sciences - Bhubaneswar

Manas Kumar Panigraphi

All India Institute of Medical Sciences - Bhubaneswar

\section{Sudipta Mohakud}

All India Institute of Medical Sciences - Bhubaneswar

\section{Research Article}

Keywords: Stomach Neoplasms, Survival, Perineural Invasion, Prognosis, Neoplasm Staging

Posted Date: January 31st, 2022

DOI: https://doi.org/10.21203/rs.3.rs-1289841/v1

License: (c) (1) This work is licensed under a Creative Commons Attribution 4.0 International License.

Read Full License 


\section{Abstract}

Background: The incidence of perineural invasion (PNI) in patients with gastric cancer (GC) is high, and patients with PNI positive disease have a poor prognosis compared to PNI negative disease. The present study aims to study the incidence and evaluate the impact of PNI on the survival outcome of a cohort of Eastern Asian GC patients.

Material and Methods: All consecutive patients undergoing curative gastrectomy were included in the study. The incidence of PNI and correlation with different clinicopathological features and overall survival was performed.

Results: 59.54\% had PNI positive disease and the median OS of PNI+ve patients was 29.3 months, while it was not reached in PNI-ve patients. The PNI positivity was a significant prognostic factor for overall survival both on univariate and multivariate analysis. On TNM-PNI staging, those with TNM stage I / II patients with PNI+ve disease had similar OS to all stage III patients $(p=0.835)$ and was worse than that of PNI-ve patients $(p<0.05)$.

Conclusion: The incidence of PNI in gastric cancer is high. The inclusion of PNI with AJCC-TNM staging may better stratify prognostic staging in curatively treated gastric cancer patients.

\section{Introduction}

Worldwide, Gastric cancer (GC) is the second most common neoplasm after colorectal cancer and is the leading cause of death due to digestive tract cancer.[1] Curative surgery with D2 lymphadenectomy is the standard approach in most treatment guidelines. Neoadjuvant/adjuvant treatment is administered to reduce recurrence and improve survival. It is essential to identify prognostic factors to select the patients better to tailor the treatments available and direct experimental approaches. [2, 3] Perineural invasion (PNI) is a process of cancer cells invading the nerves, which is also called the neurotropic carcinomatous spread. Vascular and lymphatic routes are well-documented as hallmarks of malignancy long before. However, tumor spread along the nerve has been described in the mid-1800s. PNI has recently emerged as an important and independent morphological prognostic factor in several malignancies such as pancreas, colon and rectum, prostate, head and neck, biliary tract, and stomach. Sometimes, PNI may be the only route of distant metastasis without lymphatic and vascular spread. The morphology and clinical significance of PNI are well-characterized, but its molecular mechanism is highly complex and not well elucidated.

This study aims to evaluate the impact of PNI in the long-term survival of GC patients undergoing curative surgery and discuss the impact of $\mathrm{PNI}$ on AJCC prognostic staging.

\section{Material \& Methods}


All patients who had undergone curative radical gastrectomy from 1st May 2016 to 30th December 2020 were included in the study. The data were retrieved from the prospectively maintained standalone surgical oncology database (SQL Server Express 2012/power builder 9.0). All procedures were followed according to the Institutional ethical standards and the Helsinki Declaration of 1975, as revised in 1983.

\section{Data Collection:}

The database was queried for the following data from the database (demography, location of the tumor, neoadjuvant chemotherapy, type of surgery, curative/palliative surgery, stage, histopathology, differentiation, perineural invasion, lymphovascular invasion, adjuvant therapy details, last follow-up date, any recurrence, and mortality details). The 8th edition AJCC TNM system was used for staging purposes.

\section{Inclusion and Exclusion Criteria:}

All consecutive patients who underwent curative radical gastrectomy were included in the analysis. The patients who had undergone surgery with palliative intent and those with incomplete data were excluded from the analysis.

\section{Perineural Invasion:}

The resected gastrectomy specimens received in the histopathology lab were grossed and reported as per College of American Pathologists (CAP) protocol. Hematoxylin and eosin-stained slides were prepared using a standard protocol of tissue processing. PNI status was evaluated using the criteria proposed by Batsakis JG [4]. The presence of PNI was considered when the invasion was in, around, and through the nerves. [Figure 1] Though IHCs (S100 and NSE) help pick up microscopic foci of PNI, they were not used in this study.

\section{Statistical Analysis:}

Overall survival was calculated as the time difference between the date of diagnosis and the date of death or last contact with the patient. STATA software Version 11 (Stata Corp LP, TX) was used for data analysis. Categorical data were expressed as proportion or percentage. Numerical data were expressed as mean or median (+/-SD). The difference between categorical data was compared using the Chi-squared test / Fisher's exact test. The difference between numerical data was compared using the student's t-test / ANOVA test. Survival was calculated using the Kaplan-Meier curve, and the difference in survival was compared using the log-rank test/cox proportional hazards. A p-value of $<0.05$ was considered significant.

\section{Results}

\section{Patient Characteristics:}


A total of 212 consecutive patients underwent surgery for gastric malignancy during the study period, and 131 patients met the above inclusion criteria [eFigure 1 in Supplement]. The mean age was $57.17(+/-$ 11.12) years, and the male to female ratio was $2.2: 1$.

The incidence of PNI was 78 (59.54\%) patients in this cohort. On univariate analysis, the incidence of PNI was significantly associated with location of tumour ( $74.42 \%$ in proximal, gastroesophageal junction \& diffuse tumours vs $52.27 \%$ in distal $\&$ body tumours, $p=0.015$ ); surgery type (distal gastrectomy $50 \%$ vs total gastrectomy $82.05 \%, p=0.003$ ), differentiation (well differentiated $34.62 \%$ vs moderately differentiated $53.33 \%$ vs poorly differentiated $76.79 \%, p=0.002$ ), clinical stage (early $45.10 \%$ vs locally advanced $68.75 \%, \mathrm{p}=0.007$ ), AJCC "T" stage (T1 7.69\% vs T2 $26.32 \%$ vs T3 $59.57 \%$ vs T4 $84.62 \%$, $\mathrm{p}=0.001$ ), and AJCC “ $\mathrm{N}$ " stage ( $\mathrm{N} 043.75 \%, \mathrm{vs} \mathrm{N}+64.65 \%, \mathrm{p}=0.036)$. On multivariate analysis, incidence of PNI was significantly high as the AJCC " $T$ " stage increases $(p=0.001)$ [Table 1]. 
Table 1

Demography and Patient Characteristics ( $n=131)$

\begin{tabular}{|c|c|c|c|c|c|}
\hline \multirow[t]{2}{*}{ Variable } & \multirow[t]{2}{*}{ Sub-Groups } & \multicolumn{2}{|l|}{ Incidence } & \multirow{2}{*}{$\begin{array}{l}\text { Univariate } \\
\text { Analysis } \\
\text { p-value }\end{array}$} & \multirow{2}{*}{$\begin{array}{l}\text { Multivariate } \\
\text { Analysis }\end{array}$} \\
\hline & & PNI - & PNI + & & \\
\hline \multirow[t]{2}{*}{ Age } & \multirow{2}{*}{$\begin{array}{l}\leq 50 \text { Years } \\
>50 \text { Years }\end{array}$} & $\begin{array}{l}31 \\
(39.24 \%)\end{array}$ & $\begin{array}{l}48 \\
(60.76 \%)\end{array}$ & \multirow[t]{2}{*}{0.726} & \multirow[t]{2}{*}{0.340} \\
\hline & & $\begin{array}{l}22 \\
(42.31 \%)\end{array}$ & $\begin{array}{l}30 \\
(57.69)\end{array}$ & & \\
\hline \multirow[t]{3}{*}{ Gender } & \multirow{3}{*}{$\begin{array}{l}\text { Female } \\
\text { Male }\end{array}$} & 16 & 25 & \multirow[t]{3}{*}{0.821} & \multirow[t]{3}{*}{0.516} \\
\hline & & $(09.0<10)$ & (סלק. & & \\
\hline & & $\begin{array}{l}37 \\
(41.11 \%)\end{array}$ & $\begin{array}{l}53 \\
(58.89 \%)\end{array}$ & & \\
\hline \multirow[t]{2}{*}{ Tumour Location } & $\begin{array}{l}\text { GE Junction, Proximal } \\
\text { and Diffuse }\end{array}$ & $\begin{array}{l}11 \\
(25.58 \%)\end{array}$ & $\begin{array}{l}32 \\
(74.42 \%)\end{array}$ & \multirow[t]{2}{*}{0.015} & \multirow[t]{2}{*}{0.299} \\
\hline & Body, Distal & $\begin{array}{l}42 \\
(47.73 \%)\end{array}$ & $\begin{array}{l}46 \\
(52.27 \%)\end{array}$ & & \\
\hline \multirow[t]{3}{*}{ Surgery Type } & \multirow{2}{*}{$\begin{array}{l}\text { Total Gastrectomy } \\
\text { Distal Gastrectomy }\end{array}$} & $\begin{array}{l}7 \\
(17.95 \%)\end{array}$ & $\begin{array}{l}32 \\
(82.05 \%)\end{array}$ & \multirow[t]{3}{*}{0.003} & \multirow[t]{3}{*}{0.797} \\
\hline & & $40(50 \%)$ & $40(50 \%)$ & & \\
\hline & Others & $6(50 \%)$ & $6(50 \%)$ & & \\
\hline \multirow[t]{2}{*}{ Morphology } & Proliferative & $12(60 \%)$ & $8(40 \%)$ & \multirow[t]{2}{*}{0.053} & \multirow[t]{2}{*}{0.088} \\
\hline & Non-Proliferative & $41(40 \%)$ & $70(60 \%)$ & & \\
\hline \multirow[t]{4}{*}{ Differentiation } & & $\begin{array}{l}17 \\
(65.38 \%)\end{array}$ & $\begin{array}{l}9 \\
(34.62 \%)\end{array}$ & \multirow[t]{4}{*}{0.002} & \multirow[t]{4}{*}{0.301} \\
\hline & Differentiated & $\begin{array}{l}14 \\
(46.67 \%)\end{array}$ & $\begin{array}{l}16 \\
(53.33 \%)\end{array}$ & & \\
\hline & \multirow{2}{*}{$\begin{array}{l}\text { Poorly Differentiated } \\
\text { Unknown }\end{array}$} & $\begin{array}{l}13 \\
(23.21 \%)\end{array}$ & $\begin{array}{l}43 \\
(76.79 \%)\end{array}$ & & \\
\hline & & $\begin{array}{l}9 \\
(47.37 \%)\end{array}$ & $\begin{array}{l}10 \\
(52.63 \%)\end{array}$ & & \\
\hline \multirow[t]{2}{*}{ Clinical Stage } & Early & $\begin{array}{l}28 \\
(54.90 \%)\end{array}$ & $\begin{array}{l}23 \\
(45.10 \%)\end{array}$ & \multirow[t]{2}{*}{0.007} & \multirow[t]{2}{*}{0.415} \\
\hline & & $\begin{array}{l}25 \\
(31.25 \%)\end{array}$ & $\begin{array}{l}55 \\
(68.75 \%)\end{array}$ & & \\
\hline
\end{tabular}




\begin{tabular}{|c|c|c|c|c|c|}
\hline \multirow[t]{2}{*}{ Variable } & \multirow[t]{2}{*}{ Sub-Groups } & \multicolumn{2}{|l|}{ Incidence } & \multirow{2}{*}{$\begin{array}{l}\text { Univariate } \\
\text { Analysis } \\
\text { p-value }\end{array}$} & \multirow{2}{*}{$\begin{array}{l}\text { Multivariate } \\
\text { Analysis } \\
\text { p-value }\end{array}$} \\
\hline & & PNI - & $\mathrm{PNI}+$ & & \\
\hline \multirow[t]{2}{*}{$\begin{array}{l}\text { Lymphovascular } \\
\text { Invasion }\end{array}$} & Yes & $\begin{array}{l}31 \\
(35.23 \%)\end{array}$ & $\begin{array}{l}57 \\
(64.77 \%)\end{array}$ & \multirow[t]{2}{*}{0.081} & \multirow[t]{2}{*}{0.094} \\
\hline & No & $\begin{array}{l}22 \\
(51.16 \%)\end{array}$ & $\begin{array}{l}21 \\
(48.84 \%)\end{array}$ & & \\
\hline \multirow[t]{5}{*}{ AJCC “T” Stage } & T1 & 12 & 1 & \multirow[t]{5}{*}{0.001} & \multirow[t]{5}{*}{0.002} \\
\hline & $\mathrm{T} 2$ & & & & \\
\hline & T3 & $\begin{array}{l}14 \\
\text { (73.68\%) }\end{array}$ & $\begin{array}{l}5 \\
(26.32 \%)\end{array}$ & & \\
\hline & \multirow[t]{2}{*}{$\mathrm{T} 4$} & $\begin{array}{l}19 \\
(40.43 \%)\end{array}$ & $\begin{array}{l}28 \\
(59.57 \%)\end{array}$ & & \\
\hline & & $\begin{array}{l}8 \\
(15.38 \%)\end{array}$ & $\begin{array}{l}44 \\
(84.62 \%)\end{array}$ & & \\
\hline \multirow[t]{2}{*}{ AJCC “N" Stage } & NO & $\begin{array}{l}18 \\
(56.25 \%)\end{array}$ & $\begin{array}{l}14 \\
(43.75 \%)\end{array}$ & \multirow[t]{2}{*}{0.036} & \multirow[t]{2}{*}{0.104} \\
\hline & $\mathrm{N}+$ & $\begin{array}{l}35 \\
(35.35 \%)\end{array}$ & $\begin{array}{l}64 \\
(64.65 \%)\end{array}$ & & \\
\hline \multirow{2}{*}{$\begin{array}{l}\text { Adjuvant } \\
\text { Therapy }\end{array}$} & Chemotherapy & \multirow{2}{*}{$\begin{array}{l}35 \\
(37.23 \%)\end{array}$} & \multirow{2}{*}{$\begin{array}{l}59 \\
(62.77 \%)\end{array}$} & 0.231 & 0.649 \\
\hline & Chemoradiation & & & 0.094 & - \\
\hline Recurrence & Yes & $\begin{array}{l}6 \\
(26.09 \%)\end{array}$ & $\begin{array}{l}17 \\
(73.91 \%)\end{array}$ & & \\
\hline Mortality & Yes & $\begin{array}{l}10 \\
(21.74 \%)\end{array}$ & $\begin{array}{l}36 \\
(78.26 \%)\end{array}$ & & \\
\hline \multicolumn{2}{|c|}{ Median Overall Survival (months) } & $\begin{array}{l}\text { Not } \\
\text { Reached }\end{array}$ & $\begin{array}{l}29.3 \\
\text { months }\end{array}$ & & \\
\hline
\end{tabular}

After a median follow-up of 17.7 months, 23 (17.56\%) patients had a recurrence, and $46(35.11 \%)$ patients died. The median overall survival (OS) of the cohort of 131 patients was 35.87 months. The median OS for patients with PNI+ve was 29.3 months, while the median survival was not reached for PNIve patients. The PNI was an independent prognostic factor influencing OS with the 3-year OS of $65.14 \%$ (95\% Cl 42.06-80.89) for PNI -ve vs. $32.50 \%(95 \% \mathrm{Cl} 17.90-47.99)$ for PNI+ve patients $(p=0.009)$ [Figure 2]. On the log-rank test of different subgroups, PNI positivity was significantly associated ( $p$-value $<0.05)$ with poor survival in patients with age $>50$ years, male gender, tumors in the body $\&$ antro-pyloric region of the stomach, and non-proliferative gross morphology, any clinical-stage, any lymphovascular invasion, pathological T4, pathological node-positive, and N3 disease [Figure 3], [eTable 1 in Supplement]. On 
multivariate analysis with Cox regression, PNI positivity was an independent prognostic factor (HR 2.73, 95\% Cl 1.194-6.244, $p=0.01$ ).

The patients who underwent upfront surgery (those without neoadjuvant treatment, $n=91$ ) were tested whether PNI status had any influence on survival in different subgroups (TNM stage, T stage, and N stage). The patients who underwent neoadjuvant therapy were excluded from this analysis to exclude the influence of chemotherapy on PNI in the post-chemotherapy resected specimen. In this cohort of upfront surgery patients, the PNI was incorporated to TNM staging (TNM-PNI staging). The patients with TNM stage I / II patients with PNI+ve disease had worse overall survival than that of PNI-ve patients. While the patients with TNM stage I / II disease with PNI+ve disease had similar survival to TNM stage III patients $(p>0.05)$ [Figure 2B]. The patients with stage III-PNI -ve disease had similar survival to stagel/II PNI-ve disease ( $p>0.05$ ) [Table 2]. Similarly, the T stages ( $p T 4$ vs. pT1-3, the pT1/3 group were clubbed together due to fewer patients in T1/2), and $\mathrm{N}$ stages could be prognosticated into two subgroups by PNI status. The patients with pT1-3 with PNI +ve had similar survival to patients with pT4 disease (pT4 with PNI -ve and +ve together) [Figure 2C]. Similarly, it was observed that the patients with pNO \& PNI +ve disease had worse overall survival, which was similar to patients of $\mathrm{pN}+$ disease ( $\mathrm{pN}+$ with $\mathrm{PNI}$-ve and +ve together) (p-value>0.05) [Figure 2D][6].

Table 2

AJCC TNM Stage and TNM-PNI Stage Grouping vs Hazards of Death

\begin{tabular}{|c|c|c|c|c|c|c|}
\hline \multicolumn{3}{|c|}{ AJCC TNM Staging } & \multicolumn{4}{|c|}{ TNM-PNI Grouping } \\
\hline $\begin{array}{l}\text { TNM } \\
\text { Stage }\end{array}$ & $\begin{array}{l}\text { Hazard } \\
\text { Ratio } \\
(95 \% \mathrm{Cl})\end{array}$ & $\begin{array}{l}\text { p- } \\
\text { value }\end{array}$ & TNM-PNI & $\begin{array}{l}\text { No of } \\
\text { Patients }\end{array}$ & $\begin{array}{l}\text { Hazard Ratio } \\
(95 \% \mathrm{Cl})\end{array}$ & $\begin{array}{l}\mathrm{p}- \\
\text { value }\end{array}$ \\
\hline \multirow[t]{3}{*}{ Stage I } & \multirow[t]{3}{*}{1} & \multirow[t]{3}{*}{-} & Stage I-PNI Neg & $17(18.68 \%)$ & 1 & - \\
\hline & & & \multirow[t]{2}{*}{ Stage I-PNI Pos } & \multirow[t]{2}{*}{$3(3.30 \%)$} & 4.18 & \multirow[t]{2}{*}{0.312} \\
\hline & & & & & $(0.26-66.97)$ & \\
\hline \multirow[t]{4}{*}{ Stage II } & \multirow{4}{*}{$\begin{array}{l}5.94 \\
(1.28-7.55)\end{array}$} & \multirow[t]{4}{*}{0.023} & \multirow[t]{2}{*}{ Stage II-PNI Neg } & \multirow[t]{2}{*}{$9(9.89 \%)$} & 7.48 & \multirow[t]{2}{*}{0.101} \\
\hline & & & & & $(0.67-83.01)$ & \\
\hline & & & \multirow[t]{2}{*}{ Stage II-PNI Pos } & \multirow[t]{2}{*}{$14(15.38 \%)$} & 10.49 & \multirow[t]{2}{*}{0.028} \\
\hline & & & & & $(1.29-85.44)$ & \\
\hline \multirow[t]{4}{*}{ Stage III } & \multirow{4}{*}{$\begin{array}{l}6.03 \\
(1.39-6.06)\end{array}$} & \multirow[t]{4}{*}{0.016} & \multirow{2}{*}{$\begin{array}{l}\text { Stage III-PNI } \\
\text { Neg }\end{array}$} & \multirow[t]{2}{*}{$14(15.38 \%)$} & 2.87 & \multirow[t]{2}{*}{0.389} \\
\hline & & & & & $(0.26-31.89)$ & \\
\hline & & & \multirow{2}{*}{$\begin{array}{l}\text { Stage III-PNI } \\
\text { Pos }\end{array}$} & \multirow[t]{2}{*}{$34(37.36 \%)$} & 13.52 & \multirow[t]{2}{*}{0.012} \\
\hline & & & & & $\begin{array}{l}(1.79- \\
102.10)\end{array}$ & \\
\hline
\end{tabular}




\section{Discussion}

In the present article, we studied the incidence and prognostic value of $\mathrm{PNI}$ in patients who underwent curative resection for gastric adenocarcinoma. The incidence of PNI detected by histopathology analysis after surgery was $59.54 \%$. The PNI positivity was higher in proximal, gastroesophageal junction, diffuse tumors; who had undergone total gastrectomy; less differentiated tumors; advanced disease; higher T or $\mathrm{N}$ stage. However, on multivariate analysis, only the AJCC T stage was significantly associated with a higher incidence of PNI.. The median OS of the whole cohort was 35.87 months, while that of PNI+ve was 29.3 months PNI-ve patients did not reach median survival. On univariate and multivariate analysis, the PNI positivity was a significant prognostic factor. The hazard of death in $\mathrm{PNI}+\mathrm{ve}$ disease was worse in all subgroups. However, it was significant in older than 50 years, males, tumors in the body \& antro-pyloric region of the stomach, non-proliferative gross morphology, pathological T4, pathological node-positive, and N3 disease.

The incidence of PNI in gastric cancer has been reported by various series from 31.7 - 73\% [Table 3]. Different authors have studied the factors associated with PNI positivity. (e.g., the site of the tumor, Borrmann classification, the depth of invasion (pT stage), tumor size, differentiation, lymph node metastasis ( $\mathrm{pN}$ stage), clinical stage, histological type, lymphatic vessel invasion, and blood vessel invasion, pathological stage were closely associated with increased PNI positivity). But all studies consistently showed an increase in PNI positivity rate as the AJCC TNM stage, T stage, or N stage increases. (ref) The present study also showed a similar trend in the PNI positivity rate. 
Table 3

Published Studies on Perineural Invasion in Gastric Cancer and Survival

\begin{tabular}{|c|c|c|c|c|}
\hline Author \& Year & Resection & Stages & Incidence of PNI & Survival (PNI-ve vs. PNI+ve) \\
\hline $\begin{array}{l}\text { Tanaka et al. } \\
1994[5]\end{array}$ & $\mathrm{R} 0 / \mathrm{R}+$ & I/II/III & 139/283 (49.1\%) & $\begin{array}{l}5 \text {-year survival rate } 71.3 \% \\
\text { vs. } 23.7 \%, p<0.001\end{array}$ \\
\hline $\begin{array}{l}\text { Duraker et al. } \\
2003[6]\end{array}$ & $\mathrm{R} 0 / \mathrm{R}+$ & I/II/III/IV & $211 / 354(59.6 \%)$ & $\begin{array}{l}\text { OS, HR: } 1.10(0.89-1.37) ; \mathrm{T} 3, \\
\text { HR: } 1.16(0.91-1.48)\end{array}$ \\
\hline $\begin{array}{l}\text { Scartozzi et al. } \\
2006[2]\end{array}$ & Ro & I/II/III & $211 / 287$ (73\%) & $\begin{array}{l}\text { Median survival } 38.2 \text { months vs } \\
24.1 \text { months, } p=0.008\end{array}$ \\
\hline $\begin{array}{l}\text { Tianhang et al. } \\
2008[7]\end{array}$ & $\mathrm{R} 0 / \mathrm{R}+$ & I/II/III/IV & $518 / 1,632(31.7 \%)$ & $\begin{array}{l}\text { OS, } \mathrm{HR}=3.23,95 \% \mathrm{Cl}=2.6-8.11 \\
\mathrm{p}<0.01\end{array}$ \\
\hline $\begin{array}{l}\text { Bilici et al. } 2010 \\
\text { [8] }\end{array}$ & Ro & IB/II/III/IV & 180/238 (75.6\%) & $\begin{array}{l}\text { Median OS, } 64.9 \text { vs. } 28.1 \\
\text { months, } p=0.001\end{array}$ \\
\hline $\begin{array}{l}\text { Selçukbiricik et } \\
\text { al, 2012 [9] }\end{array}$ & Ro & I/II/III & $211 / 287(73 \%)$ & $\begin{array}{l}\text { Median OS, } 38.2 \text { vs. } 24.1 \\
\text { months, } p=0.008\end{array}$ \\
\hline $\begin{array}{l}\text { Jiang et al. } 2014 \\
\text { [10] }\end{array}$ & Ro & $\mathrm{I} / \mathrm{II} / \mathrm{III}$ & 186/518 (35.9\%) & $\begin{array}{l}\text { OS, } \mathrm{HR}=1.901,95 \% \mathrm{Cl}=1.495- \\
2.418, \mathrm{P}<0.001\end{array}$ \\
\hline \multirow[t]{2}{*}{$\begin{array}{l}\text { Aurello et al. } \\
2017 \text { [3] }\end{array}$} & Ro & $\mathrm{Ib} / \mathrm{II} / \mathrm{III}$ & $47 / 103(45.6 \%)$ & $\begin{array}{l}\text { 5-year actuarial OS (59\% vs } 19 \% \text {, } \\
P=0.0001) \text { and }\end{array}$ \\
\hline & & & & DFS ( $69 \%$ vs $22 \%, P=0.0001)$ \\
\hline $\begin{array}{l}\text { De Franco et al. } \\
2018[11]\end{array}$ & $\mathrm{R} 0 / \mathrm{R}+$ & I/II/III/IV & 162/455 (33.9\%) & $\begin{array}{l}\text { 5-year cancer-related survival } \\
\text { (CRS) was } 65.7 \% \text { vs. } 20.6 \%, p< \\
0.001\end{array}$ \\
\hline Our series, 2021 & Ro & I/II/III & 78/131 (59.54\%) & $\begin{array}{l}\text { 3-year OS rate } 65.14 \% \text { vs. } \\
32.50 \%, 0.009\end{array}$ \\
\hline
\end{tabular}

Different authors have studied the prognostic role of PNI in GC patients. $[2,3,5,6,6-9,11]$ The survival has consistently been reported to be significantly worse in PNI+ve patients than PNI -ve. [Table 3] In a study by Aurello et al. from Italy, PNI could effectively differentiate locally advanced gastric cancer into two statistically significant groups demonstrating different recurrence rates and survival. [3] In another study by Scartozzi et al., early gastric cancer can be effectively differentiated into biologically two prognostic groups.[2] Two meta-analyses published in 2008 and 2020 have shown a worse survival in PNI positive patients. $[12,13]$ The present study showed a worse survival in early gastric cancer (stage I/II) when PNI was positive. Thus, the presence of PNI can now be accepted as a poor prognostic factor in gastric cancer patients.

Several studies have demonstrated that incorporation of PNI in the TNM staging gives a more accurate prognostic group. $[10,14,15]$ As per the data from previous studies and our analysis, PNI can effectively differentiate AJCC stage groups into PNI negative as a "good" and PNI positive as a "poor" prognostic 
group. Attempts should be made to analyze large institutional / multi-institutional gastric cancer databases and validate the incorporation into the UICC AJCC staging system. Patients with PNI may be candidates for aggressive oncologic and experimental therapy. In addition, the role of PNI in conventional and experimental therapy may be established.

Limitations:

The limitations of the present study were the retrospective design and a relatively small sample size. Therefore, a large-scale prospective study is needed to confirm the results.

\section{Conclusion}

In summary, Perineural Invasion is an independent marker of poor prognosis in patients with gastric cancer. The presence of Perineural Invasion identifies poor-risk patients after curative gastrectomy who are at high risk of recurrence and worse survival. Those without Perineural Invasion have a better prognosis. Incorporating Perineural Invasion into TNM staging (TNM-PNI) may better prognosticate gastric cancer patients than the 8th edition of the AJCC staging system.

\section{Declarations}

\section{Acknowledgment:}

We acknowledge the contribution of Mr. Tushar Ranjan Pradhan in designing the surgical oncology database and Ms Rukchhanda Nayak, Mr Prakash Palei for data keeping and retrieval.

Funding: No funding received

Conflict of Interest: The authors declare that they have no conflict of interest.

\section{References}

1. Ferlay J, Ervik M, Lam F, Colombet M, Mery L, Piñeros M, Znaor A, Soerjomataram I, Bray F. (2020). Global Cancer Observatory: Cancer Today. Lyon, France: International Agency for Research on Cancer. Available from: https://gco.iarc.fr/today, accessed [16 Sept 2021].

2. Scartozzi M, Galizia E, Verdecchia L, et al. Lymphatic, blood vessel and perineural invasion identifies early-stage high-risk radically resected gastric cancer patients. Br J Cancer. 2006;95:445-9. https://doi.org/10.1038/sj.bjc.6603286.

3. Aurello P, Berardi G, Tierno SM, et al. Influence of perineural invasion in predicting overall survival and disease-free survival in patients With locally advanced gastric cancer. Am J Surg. 2017;213:748-53. https://doi.org/10.1016/j.amjsurg.2016.05.022.

4. Batsakis JG. Nerves and neurotropic carcinomas. Ann Otol Rhinol Laryngol. 1985;94:426-7. 
5. Bilici A, Seker M, Ustaalioglu BBO, et al. Prognostic significance of perineural invasion in patients with gastric cancer who underwent curative resection. Ann Surg Oncol. 2010;17:2037-44. https://doi.org/10.1245/s10434-010-1027-y.

6. De Franco L, Marrelli D, Voglino C, et al. Prognostic Value of Perineural Invasion in Resected Gastric Cancer Patients According to Lauren Histotype. Pathol Oncol Res. 2018;24:393-400. https://doi.org/10.1007/s12253-017-0257-8.

7. Duraker N, Sişman S, Can G. The significance of perineural invasion as a prognostic factor in patients with gastric carcinoma. Surg Today. 2003;33:95-100. https://doi.org/10.1007/s005950300020.

8. Selçukbiricik F, Tural D, Büyükünal E, Serdengeçti S. Perineural invasion independent prognostic factors in patients with gastric cancer undergoing curative resection. Asian Pac J Cancer Prev. 2012;13:3149-52.

9. Tanaka A, Watanabe T, Okuno K, Yasutomi M. Perineural invasion as a predictor of recurrence of gastric cancer. Cancer. 1994;73:550-5. https://doi.org/10.1002/10970142(19940201)73:3<550::AID-CNCR2820730309>3.0.CO;2-0.

10. Tianhang L, Guoen F, Jianwei B, Liye M. The effect of perineural invasion on overall survival in patients with gastric carcinoma. J Gastrointest Surg. 2008;12:1263-7. https://doi.org/10.1007/s11605-008-0529-4.

11. Deng J, You Q, Gao Y, et al. Prognostic value of perineural invasion in gastric cancer: a systematic review and meta-analysis. PLoS One. 2014;9:e88907. https://doi.org/10.1371/journal.pone.0088907.

12. Zhao B, Lv W, Mei D, et al. Perineural invasion as a predictive factor for survival outcome in gastric cancer patients: a systematic review and meta-analysis. J Clin Pathol. 2020;73:544-51. https://doi.org/10.1136/jclinpath-2019-206372.

13. Gao Z-M, Wang R-Y, Deng P, et al. TNM-PNI: a novel prognostic scoring system for patients with gastric cancer and curative D2 resection. CMAR. 2018;10:2925-33. https://doi.org/10.2147/CMAR.S169206.

14. Jiang N, Deng J-Y, Liu Y, et al. Incorporation of perineural invasion of gastric carcinoma into the 7th edition tumor-node-metastasis staging system. Tumour Biol. 2014;35:9429-36. https://doi.org/10.1007/s13277-014-2258-5.

15. Jung DH, Kim J-H, Lee YC, et al. Is the 7th TNM edition suitable for biological predictor in early gastric cancer? Hepatogastroenterology. 2013;60:1225-30. https://doi.org/10.5754/hge11579.

\section{Figures}

\section{Figure 1}

Histopathological Diagnosis of Perineural Invasion 
Figure $1 \mathrm{~A}$. Tumor cells around the nerve bundle covering more than $2 / 3^{\text {rd }}$ of the circumference, Figure $1 \mathrm{~B}$. Tumor cells in the perineural space (arrow), Figure 1C. Tumor cells invading the nerve bundle with acinar differentiation (yellow star), Figure 1D. Tumor cells and extracellular mucin invading the nerve bundle (green star), with surrounding inflammatory cells (arrow)

\section{Figure 2}

Kaplan-Meier Estimate of Overall Survival

Figure 2A: based on PNI (Perineural Invasion) Status, Figure 2B, 2C \& 2D: Kaplan-Meier Survival Curve of patients who have undergone upfront surgery, Figure 2B: Overall Survival of Stage I/II with and without PNI vs Stage III, Figure 2C: Overall Survival of T1/2/3 with and without PNI vs T4, Figure 2D: Overall Survival of Node Negative Disease with and without PNI vs Node Positive Disease

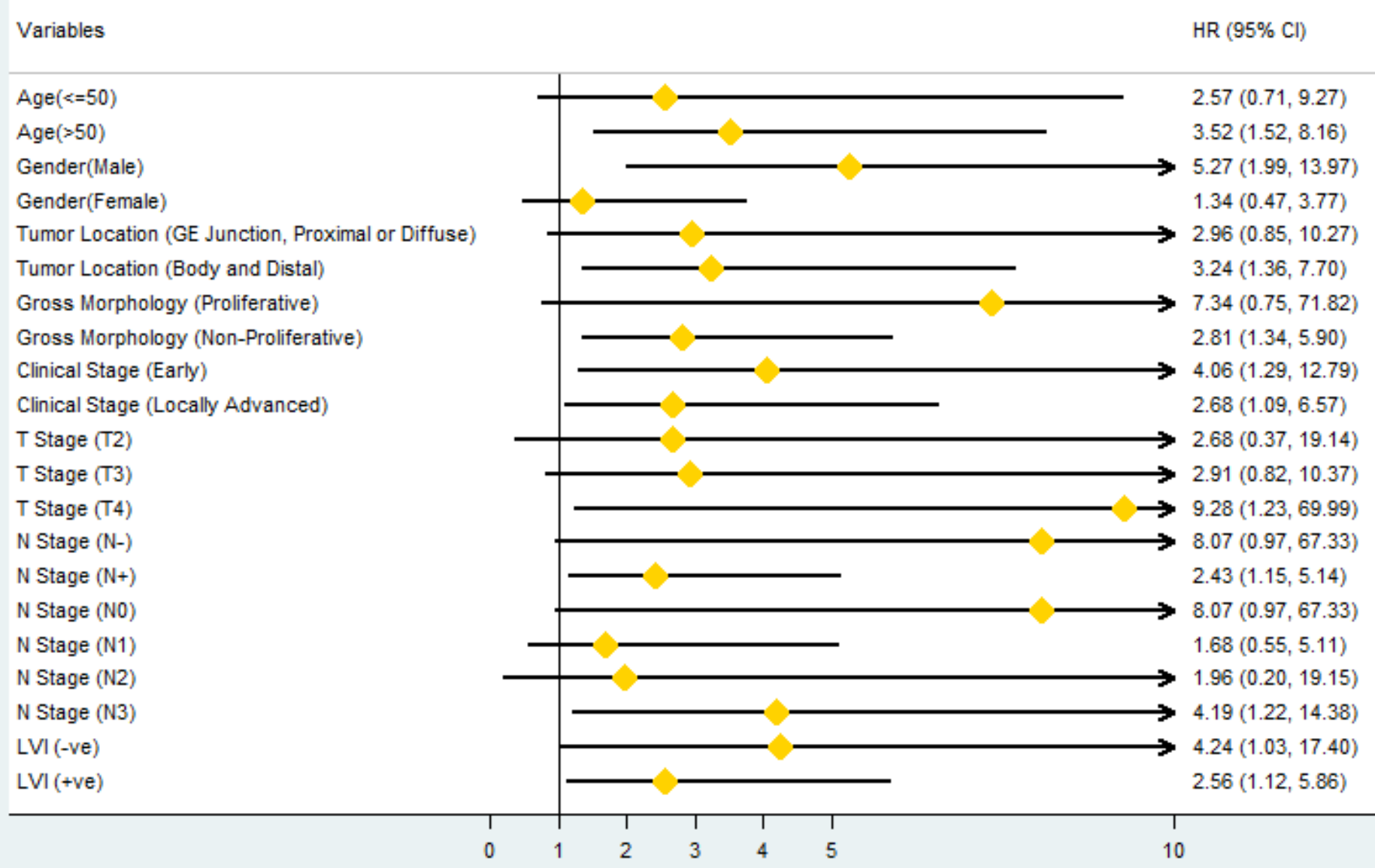

Figure 3 
Forrest Plot Hazard Ratio with different variables of the population

\section{Supplementary Files}

This is a list of supplementary files associated with this preprint. Click to download.

- eFigure1inSupplement.tiff

- eTable1inSupplement.docx 